Meta

Journal des traducteurs

Translators' Journal

\title{
Le miroir aux alouettes en intelligence artificielle
}

\section{Jean-Claude Boulanger}

Volume 32, numéro 3, septembre 1987

La fertilisation terminologique dans les langues romanes

URI : https://id.erudit.org/iderudit/002121ar

DOI : https://doi.org/10.7202/002121ar

Aller au sommaire du numéro

Éditeur(s)

Les Presses de l'Université de Montréal

ISSN

0026-0452 (imprimé)

1492-1421 (numérique)

Découvrir la revue

Citer cet article

Boulanger, J.-C. (1987). Le miroir aux alouettes en intelligence artificielle. Meta, 32(3), 326-331. https://doi.org/10.7202/002121ar d'utilisation que vous pouvez consulter en ligne.

https://apropos.erudit.org/fr/usagers/politique-dutilisation/ 


\section{LE MIROIR AUX ALOUETTES EN INTELLIGENCE ARTIFICIELLE}

JEAN-Claude Boulanger

Université Laval, Québec, Canada

La décision de fonctionner dans sa langue maternelle ou dans une langue "nationale " (en élaborant des terminologies) ou de se résigner à emprunter la langue outil avec la matière à travailler est politique, car toute langue est capable de tout nommer : l'impression trop fréquente que certaines sémantiques ne peuvent répondre au besoin notionnel relève de l'idéologie (Rey $1979: 66-67$ ).

\section{INTRODUCTION}

Le présent exposé prend sa source dans deux séminaires de $2^{\mathrm{e}}$ et $3^{\mathrm{e}}$ cycles consacrés à la néologie et tenus respectivement à l'Université Laval et à l'Université du Québec à Chicoutimi de janvier à avril 1986 sur le thème de l'emprunt dans le domaine de l'intelligence artificielle ${ }^{1}$. Seize étudiants ont participé aux rencontres (12 à Québec et 4 à Chicoutimi). Ils devaient réaliser une recherche appliquée qui consistait à extraire d'une documentation récente les termes d'emprunt appartenant au secteur de l'IA. Chaque étudiant avait à traiter dix notions, une notion pouvant donner lieu à plus d'un emprunt, ce qui occasionnait des cas de synonymie (ex. bottom up method et bottom up system). L'exercice s'appuyait sur les principes et méthodes mis au point au Québec pour le traitement des néologismes (voir Boulanger 1979). Outre le traitement terminologique habituel, les participants avaient comme mission d'établir le statut lexicographique, terminographique et informaticographique des unités terminologiques recensées. De plus, ils avaient l'obligation de se prononcer sur le statut normatif des emprunts, c'est-à-dire que pour chaque terme étranger retenu, il fallait décider de son intégration en français ou de son remplacement par une forme lexicale de facture "bien française". Ce dernier exercice plaçait les étudiants en situation réelle d'interventionnisme linguistique puisque la décision de retenir ou de rejeter l'emprunt leur revenait et qu'elle devait être justifiée.

Le groupe étant composé de linguistes, de terminologues et de traducteurs, les attitudes devant l'emprunt ont varié en fonction des critères de formation de chacun ainsi que de leur conception réciproque de la norme lexicale en français. Au surplus, l'expérience se déroulant au Québec où les comportements devant l'emprunt diffèrent profondément de ceux que l'on observe en France, il fallait s'attendre à des prises de position favorables à la francisation intégrale des termes allogènes, du moins en apparence. En fait, les Québécois souscrivent à l'hypothèse qui soutient que certaines communautés " rebelles à l'emprunt pur et simple pratiquent plus volontiers le calque qui transpose dans leur système de formation un dérivé ou composé d'une autre langue " (Bonnard $1972: 1580$ ).

$\mathrm{Au}$ total, 101 termes anglais ont été recueillis, y compris quelques variantes morphologiques ou orthographiques. Ils représentent environ 80 notions différentes. Ils ont donné lieu à une cueillette de 212 termes "français" répartis comme suit :

45 emprunts complets ou intégraux (xénotermes);

- 63 calques complets ou intégraux ; 
- 61 calques partiels ou hybrides;

43 termes français autochtones (endotermes).

Parmi les 124 calques, 19 conservent des traces visibles d'un élément étranger que ce soit un emprunt déjà intégré (compteur de bits), un calque morphologique (vecteur de mappage, résolveur de problème), un nouvel emprunt (programme bootstrap), ou encore une forme fautive (signal digitalisé).

L'hypothèse de départ cherchait à vérifier si l'intrusion des techniques et technologies américaines récentes dans la vie des francophones avait pour effet immédiat d'accroître le nombre de vocables empruntés en même temps que les réalias qu'ils désignent. De vérifier également si le calque pouvait constituer une caractéristique des terminologies nouvelles dans une langue, surtout lors de la phase d'installation.

D'où la mise en évidence de trois problèmes linguistiques majeurs, parmi d'autres :

1. L'impossibilité ou la quasi-impossibilité d'harmoniser les terminologies au plan international, c'est-à-dire de les normaliser.

2. La mise en relief du facteur de la synonymie avec les avantages et les inconvénients qui en découlent, comme par exemple les flottements terminologiques presque permanents.

3. La perturbation des systèmes linguistiques, tout spécialement les zones lexicales, morphosémantiques et morphosyntaxiques des langues non productrices de la technologie nouvelle. Le plus souvent ces langues empruntent massivement à des systèmes allogènes d'où émane la terminologie. En conséquence, elles ne sont pas immunisées convenablement contre l'invasion. Quand elles n'empruntent pas, elles calquent, émaillant le langage de fleurs exotiques souvent empoisonnées.

Toutes les innovations nécessitent l'élaboration et la mise en service constantes de terminologies scientifiques et techniques nouvelles en vue d'assurer l'efficacité de la communication ainsi que la diffusion des produits et des informations. La floraison lexicale concourt à l'inflation souvent excessive des dictionnaires chargés d'en rendre compte, en particulier les dictionnaires terminologiques et les banques de terminologie. Les termes voyagent non seulement dans leur langue d'origine mais ils émigrent dans d'autres langues suivant des modalités variables. Tantôt ils y arrivent intacts, tantôt ils s'y faufilent sous la forme d'une construction savamment maquillée ou encore légèrement vêtus d'attributs naturels. "La découverte des réalités d'un pays étranger passe presque toujours par la langue étrangère correspondante, car dans un premier temps on ne peut en parler qu'en les appelant par leur nom "(Rey-Debove/Gagnon 1980 : XI). Le xénoterme précède souvent le calque ou le néologisme originaire, la nécessité de dénommer pressant davantage que la mise au point d'un vocable conforme aux critères de la langue. Des mots allogènes se sont vu ensuite supplantés par des nouveautés internes (ex. logiciel), d'autres ont résisté sous la forme étrangère (ex. wetware) ou calquée (ex. signal numérique), tandis que d'autres enfin vivent en concubinage avec un ou des synonymes internes (ex. matching score / évaluation de confirmation ; pattern matching / filtrage / appariement).

Tout comme l'emprunt, le calque est simultanément un procédé et le résultat de l'opération. Il répond donc à des besoins de dénomination tout en s'inscrivant comme un mode organique de formation d'une langue à des gènes qui se combinent, on peut penser que le calque s'apparente à une manipulation génétique, qu'il est le fruit d'une espèce de mutation morphosyntaxique artificielle dans une langue. (Ce qui vient d'être dit vaut uniquement pour le calque formel, car le calque sémantique n'entre pas dans nos préoccupations pour le moment.) 
Le calque procédé sera défini comme étant la substitution d'un signifiant de texture étrangère par un signifiant ancien ou nouveau de texture nationale pour désigner soit un signifié totalement étranger, soit un signifié déjà opérationnel en français, soit un signifié lui-même nouveau en français.

Quant au calque-produit, c'est l'unité lexicale issue de la substitution.

Le calque se situe juste à l'intersection des moyens externes (emprunt) et des moyens internes (dérivation, composition, etc.) de renouvellement du stock lexical d'une langue. On le considère comme un xénomorphème (simple ou complexe) transposé d'une langue dans une autre, façonné dans la matrice morphosyntaxique de la langue d'accueil et accompagné ou non de bon sens dans la langue source.

Manifestement, le calque provient du contact entre deux langues et, dans la première phase de son installation, il doit beaucoup au bilinguisme actif ou passif du calqueur. Si l'action de calquer exige une certaine forme de bilinguisme, la réutilisation du calque intégré n'y est pas obligatoirement rattachée. Il y a donc calque lorsqu'un xénomorphème est cloné dans un système linguistique différent, manipulé par des locuteurs qui ne sont jamais ou rarement en situation de communication bilingue. Au fur et à mesure que la motivation d'emprunt ou de calque s'estompe, les interférences ne sont plus perçues (ex. est-allemand, nord-américain ; base de faits, moteur d'inférence) ; d'autant que les nouveaux termes viennent s'insérer dans des suites paradigmatiques qui sont d'allure bien française. Le calque s'apparente à une démarche interprétative. Reflétée à travers un miroir langagier, la forme étrangère reste aux portes du français, son ombre seule franchissant le seuil. Tout comme le reflet d'un objet dans un miroir n'est pas cet objet, le calque est un emprunt sans être l'emprunt. Pour les traducteurs et les terminologues, c'est un vrai miroir aux alouettes lexicales.

Dans la pratique, le calque s'avère difficile à épingler. Seule une double recherche terminologique, une dans chaque langue, permet de rassembler suffisamment d'indices pour en retracer l'origine. C'est le recoupement des informations terminologiques ou la fusion des nomenclatures qui accrédite la filiation entre le terme de départ et le terme d'arrivée. Il arrive souvent que le terminologue n'ait qu'une suspicion du calque s'il ne repère pas la matrice allogène (ex. démon, coquille, scénario, fenêtre forment en apparence des néologismes sémantiques ou des homonymes en français alors qu'ils proviennent respectivement des termes anglais demon, shell, script et window).

\section{LA XÉNOTERMINOLOGIE}

La matrice allogène du calque est un moule souple qui permet une réfection autochtone du produit fini à partir des règles propres de la langue cible, que ce soit du point de vue phonétique, sémantique, morphologique ou syntaxique. (Seuls les deux derniers volets ont retenu l'attention pour l'analyse.)

L'étude du corpus confirme que les normes pour calquer ont été respectées.

- Aucune forme française ne reprend la structure étrangère dã $\rightarrow$ dé qui se retrouve par exemple dans nord-américain (angl. north-american).

- À l'exception des emprunts directs, tous les termes obéissent au modèle français dé $\rightarrow$ dã : ex.

(angl.) combinatorial explosion backward chaining

Traduction littérale : ex.

(angl.) degree of freedom expert system

- Traduction ou adaptation partielle : ex.

(angl.) graphic package

mapping vector (fr.) explosion combinatoire ; chaînage arrière.

(fr.) degré de liberté ; système expert.

(fr.) package graphique ; vecteur de mappage. 
- Adaptation morphologique : ex.

(angl.) case grammar conceptual dependency

Adaptation syntaxique : ex.

(angl.) knowledge engineering inference engine

Adaptation morphosyntaxique : ex. (angl.) anchor point base of facts

1. Création nouvelle : ex.

(angl) adaptive robot instantiation graphic package

in Transformation par degré : ex. (angl.) expert system knowledge base inference engine knowledge engineer (fr.) grammaire casuelle ; dépendance conceptuelle.

(fr.) ingénierie de la connaissance ; moteur d'inférence.

(fr.) point d'ancrage ; base factuelle.

(fr.) adaptativité ; instanciation ; graphiciel.

(fr.) système expert $\rightarrow$ SE base de règles $\rightarrow \mathrm{BR}$ moteur d'inférence $\rightarrow$ MI ingénieur de la connaissance $\rightarrow$ ingénieur cogniticien $\rightarrow$ cogniticien

Emprunt du sigle étranger pour une désignation française : ex.

(angl.) KLIPS $\rightarrow$ (fr.) kilo-inférence logique par seconde $\rightarrow$ KLIPS

(angl.) $M P P \rightarrow$ (fr.) processeur à parallélisme massif $\rightarrow$ MPP

(angl.) $S I F T \rightarrow$ (fr.) technique simplifiée de filtrage inverse $\rightarrow$ SIFT

Sur un total de 212 termes "français", le corpus inclut 124 calques complets ou partiels. De ce nombre, 17 proviennent de propositions de remplacement faites par les étudiants ; les autres unités furent récoltées dans la documentation dépouillée ou dans les réservoirs terminographiques existants. Les suggestions étudiantes ressortent d'une opération d'aménagement linguistique, tandis que les 107 termes restants sont des calques spontanés nés dans les milieux spécialisés. Le calque volontaire dérive d'une réflexion linguistique menée sur l'emprunt par le calqueur qui connaît et maîtrise l'arsenal de règles morphosyntaxiques de la langue d'arrivée. Il utilise ces règles dans le cadre d'une opération d'aménagement terminologique dont l'objectif est la francisation.

Certains calques n'ont pas été admis par les aménageurs étudiants. Ceux-ci ont alors mis d'autres ressources néologiques naturelles à contribution pour proposer des unités lexicales, notamment la dérivation syntagmatique interne. De fait, 19 innovations nationales ont été proposées. Elles renvoient à 14 notions. Dans cet ensemble, 12 suggèrent le remplacement de calques seuls, 3 d'emprunts directs seuls et 4 de dénominations multiples (calques et emprunts).

D'un tout autre point de vue, l'interventionnisme linguistique contribue parfois à entretenir un flottement terminologique synonymique. Ainsi, la notion "knowledge engineer » est rendue par 7 dénominations françaises différentes : 2 calques complets repérés dans la documentation (ingénieur de la connaissance et ingénieur du savoir), 2 propositions de calques partiels (ingénieur cogniticien et informaticien de la connaissance), 3 formes d'origine interne, dont 2 équivalents proposés (informaticien cognitif et cogniticien), l'autre unité provenant d'une banque de terminologie (technicien d'un secteur de matière grise). Quant aux notions " expert system " et "inference engine ", elles donnent respectivement lieu à 8 et à 12 appellations françaises, de quoi encourager les fervents de la synonymie. 


\section{LE MIROIR}

Depuis Nicole Oresme au XIVe siècle jusqu'aux voyageurs en Concorde au XXe siècle, chaque époque a vu la pénétration profonde d'emprunts en français ; parmi ceux-ci, un nombre considérable de calques appelés aussi translation (voir Dubois $1963: 10$ ), se sont installés. "Sous sa forme spontanée, l'emprunt n'est pas l'aventure fortuite qui déstabilise, mais une des conditions mêmes de la vie des langues, du fait qu'elles sont parlées par des sociétés humaines, nécessairement en contact dans l'espace et dans le temps » (Hagège $1983: 61$ ). On ne peut donc songer à chasser systématiquement tous les mots étrangers afin de leur substituer des unités lexicales autochtones. Cela relèverait du plus pur donquichottisme ou encore d'une nouvelle croisade linguistique.

D'autant plus qu'il n'est pas interdit de se poser la question de savoir si les calques et les emprunts en général sont de véritables transpositions ou si parfois le locuteur n'a pas affaire à des parallélismes de construction, c'est-à-dire à des développements indépendants et souvent simultanés dans les langues en présence. Il est très fréquent que de tels développements reposent sur des virtualités morphosyntaxiques communes à deux ou plusieurs langues, moyennant parfois quelques ajustements mineurs. " Plus les similitudes entre les deux systèmes linguistiques sont nombreuses et profondes - le cas des langues apparentées généalogiquement - plus grandes seront les difficultés pour reconnaître ce qui est un emprunt [ou un calque] et ce qui ne l'est pas "(Goldis 1976:100). Par exemple, le français et l'anglais, sans être généalogiquement parents, disposent néanmoins d'un héritage commun dont les traces remontent au Moyen Âge. Sans oublier non plus que dans les sphères scientifiques et techniques, le recours à la formation gréco-latine est usuel. Ainsi, comment détecter que critérialité, déclaratif, procédural et heuristique doivent leur existence aux formes anglaises criteriality, declarative, procedural et heuristic si on ne remonte pas à la source d'inspiration. Même là, les fondements étymologiques communs laissent l'interprète perplexe.

Si l'on introduit dans le débat le critère du prestige des langues, on s'aperçoit rapidement que c'est la différence d'attrait entre deux langues (la prêteuse et l'emprunteuse) qui explique l'aspect dynamique du phénomène du calque. Celui-ci met en cause des systèmes linguistiques à travers le prestige culturel de chacun et à travers leur poids réciproque dans l'univers contemporain. S'il n'y a pas d'intervention rigoureuse, les cultures et les technologies dominées sont condamnées à l'emprunt linguistique, avec ou sans certificat de citoyenneté ou de naturalisation pour les nouveaux venus. Il est clair que "la valeur attribuée au mot emprunté est une question sociale et nationale; selon que l'idiome et le peuple auxquels on fait des emprunts sont regardés comme inférieurs ou supérieurs, ces emprunts descendent ou montent en dignité" (Nyrop 1913 : 142). Le calque est le reflet définitif et insidieux des influences qu'ont un peuple et une culture sur d'autres. Il s'inscrit dans une lutte de pouvoir. En tant que ressource linguistique innovatrice, il est " le lieu où doivent définir leurs rapports la langue et la culture, une langue de départ et une langue d'arrivée, le mot et le concept, pour savoir à quelle théorie du langage on se réfère" (Meschonnic $1976: 65$ ).

Le calque sédimente un simulacre de vérité dans la langue d'arrivée. C'est une espèce de bébé-éprouvette : une fertilisation terminologique artificielle. Qu'on ait donc affaire à l'emprunt direct ou à la translation, il reste toujours quelque chose de la langue de départ dans la langue cible, un sentiment d'extranéité.

De par sa motivation psychologique, le calque peut parfois se confondre avec un moyen de défense nationaliste. Comme la référence étymologique allogène se perd pour être remplacée par une fausse référence interne, il crée une impression de fausse sécurité linguistique. Le calqueur s'imagine rejeter ce qui lui est étranger linguistiquement en faisant appel à des éléments morphosyntaxiques internes. Dans le cas des entorses, la 
nouvelle règle s'additionne au réseau indigène déjà disponible (ex. dã $\rightarrow$ dé dans la structure : point cardinal + adjectif ou nom ethnique). Le calque parfait est donc un xénoterme qui, pour se conformer à la langue preneuse, adopte une physionomie propre à celle-ci. Les nouvelles formations acquérant une collaboration française par mimétisme, elles se confondent avec celles que l'usager est habitué de manipuler. En fait, il est plus facile de s'adapter au calque, donc de l'avaliser comme ressource créatrice, que de recourir à l'emprunt brut. Le calque s'enfouit alors dans la mémoire collective séculaire des francophones car il correspond à des habitudes langagières communautaires. Tandis que l'emprunt complet ou partiel conserve son apparence parasitaire.

Le calque entraîne une modification de la perception par rapport au référent ou encore une reconsidération de la valeur connotative du signe. L'enveloppe formelle devenue française charme le référent qui peut être lui aussi perçu comme français d'origine. Le facteur psychologique joue un rôle important dans la perception non seulement du signe mais également de l'univers. Une collection de signes étrangers passe dans une autre langue sous une forme déguisée, traînant dans son sillage une collection de notions qui peuvent ou bien enrichir des connaissances culturelles, scientifiques et technologiques ou, au contraire, les perturber.

Il n'en reste pas moins que le calque découle d'une décision interne, que ce ne sont pas les étrangers qui calquent mais bien les francophones eux-mêmes lorsque c'est la langue française qui est en cause. Avant de porter des accusations d'invasion à gauche et à droite, avant de jeter l'anathème, peut-être faudrait-il se regarder dans un miroir afin de se percevoir tel que l'on est.

Note

1. Le texte intégral de la communication a été publié dans une autre revue de linguistique. Une bibliographie plus étendue ainsi que les tableaux dans lesquels les termes sont classés par catégorie l'accompagnent.

\section{BIBLIOGRAPHIE}

BONNARD, Henri (1972) : "L'emprunt", Grand Larousse de la langue française, t. 2, Paris, Librairie Larousse, pp. 1579-1590.

BOULANGER, Jean-Claude (1979) : "Problématique d'une méthodologie d'identification des néologismes en terminoloige ", Néologie et lexicologie. Hommage à Louis Guilbert, coll. "Langue et langage", Larousse université, Paris, Librairie Larousse, pp. 36-46.

DUBOIS, Jean (1963) : "L'emprunt en français ", l'Information littéraire, no 15, pp. 10-16.

GOLDIS, Ana (1976) : "Calque linguistique' dans le cadre du contact entre deux langues apparentées : le français et le roumain " Cahiers de lexicologie, $\mathbf{n}^{\circ} 28$, fasc. 2, pp. 99-119.

HAGĖGE, Claude (1983) : "Voies et destins de l'action humaine sur les langues ", Réforme des langues. Histoire et avenir, vol. 1, Hambourg, Buske Verlag Hamburg, pp. 11-68.

MESCHONNIC, Henri (1976) : "Le calque dans la traduction", Cahiers internationaux du symbolisme, $\mathrm{n}^{\circ} 31-32$, pp. $65-75$.

NYROP, Kristofer (1913) : Grammaire historique de la langue française, t. 4, "Sémantique ", Copenhague, Gyldendalske Boghandel Nordisk Forlag, viii +496 p.

REY, Alain (1979) : la Terminologie : noms et notions, "Que sais-je?", $\mathrm{n}^{\circ} 1780$, Paris, PUF, $128 \mathrm{p}$.

REY-DEBOVE, Josette et Gilberte GAGNON (1980) : Dictionnaire des anglicismes. Les mots anglais et américains en français, "Les usuels du Robert ", Paris, le Robert, $\mathrm{xx}+1153$ p. 